\title{
QUOTIENT SHEAVES AND VALUATION RINGS
}

\author{
BY \\ JOEL CUNNINGHAM( ${ }^{1}$
}

\begin{abstract}
In this paper a construction of a quotient sheaf of a sheaf of rings is given. This construction is analogous to the Utumi ring of quotients of a ring. For a valuation ring $V$, a sheaf of rings corresponding to $V$ is introduced and its quotient sheaf is computed. It is shown that this quotient sheaf corresponds to the completion of $V$ in case $V$ is discrete rank one and that $V$ is maximal if and only if its associated sheaf of rings is its own quotient sheaf.
\end{abstract}

Introduction. The first section of this paper defines and discusses dense extensions of modules over a sheaf of rings and the quotient sheaf of a sheaf of rings. Given a sheaf $A$ of rings, a maximal dense extension of $A$ as an $A$-Module is seen to be naturally a sheaf of rings extending $A$. We call this new sheaf the quotient sheaf of $A$ and denote it as $Q(A)$. This idea of the quotient sheaf is thus analogous to the Utumi (maximal) ring of quotients of a ring. Gabriel's thesis [2] is used to give other characterizations of $Q(A)$.

In the second section we start with an arbitrary valuation ring $V$ and form a corresponding sheaf of rings $A_{V}$. This is not the usual structure sheaf for $V$; in fact its underlying topological space is the set of all divisorial ideals of $V$ rather than the set of prime ideals. We compute the quotient sheaf $Q\left(A_{V}\right)$. From this computation we see that if $V$ is discrete rank one and $W$ is the completion of $V$ then $Q\left(A_{V}\right)=A_{W}$, so that in this case the quotient sheaf gives exactly the same information as the classical completion. On the other hand if $V$ is any valuation ring, $A_{V}=Q\left(A_{V}\right)$ if and only if $V$ has no proper immediate extensions.

Throughout this paper ring means associative ring with identity and all ring homomorphisms and modules are unital.

I would like to thank D. K. Harrison and Hoyt Warner for their many helpful comments and suggestions on this work.

1. Dense extensions and the quotient sheaf. Throughout this section we let $A$ be a sheaf of rings over a topological space $X$ and let $\mathscr{M}$ be the category of (right-) $A$-Modules. Thus $\mathscr{M}$ is an abelian category with injective envelopes. For $M$ and $N$ in $\mathscr{M}, M \leqq N$ will denote that $M$ is a subobject of $N$.

Received by the editors February 25, 1971.

AMS 1970 subject classifications. Primary 18F20, 13A15; Secondary 16A08.

Key words and phrases. Sheaf of rings, sheaf of modules, dense extension, quotient sheaf, valuation ring, divisorial ideal, maximal valuation ring.

(1) This work is, in part, from the author's Ph.D. thesis written at the University of Oregon under the direction of D. K. Harrison. The author was supported at Oregon by an NSF traineeship.

Copyright (C) 1972, American Mathematical Society 
Definition. If $M \leqq N$ in $\mathscr{M}$ we say that $M$ is dense in $N$ and write $M \triangle N$ in case for all $L$ in $\mathscr{M}$ such that $M \leqq L \leqq N$ Hom $_{\mathscr{M}}(L / M, N)=0$.

This notion of dense extensions corresponds to the notion of rational extensions of modules over a ring (e.g. [1, p. 58]), and the following two results are exact analogues of the ring situation. However we do not have a characterization of density in terms of elements, so proofs somewhat different than those in [1] are needed.

Proposition 1.1. Let $L, M$ and $N$ in $\mathscr{M}$. (a) If $M \triangle N$ then $M$ is essential in $N$.

(b) If $M \triangle N$ and $L \leqq N$ then $(M \cap L) \triangle L$.

(c) If $L \Delta M$ and $M \Delta N$ then $L \Delta N$.

(d) If $M \triangle N$ and $L \triangle N$ then $(M \cap L) \triangle N$.

(e) If $M \Delta N$ and $M \leqq L \leqq N$ then $M \Delta L$ and $L \Delta N$.

(f) If $M \leqq N \leqq I$ where I is an injective envelope of $N$ then $M \Delta N$ if and only if for any $f \in \operatorname{Hom}(I, I), f(M)=0$ implies $f(N)=0$.

Proof. (a) If $0 \neq K \leqq N$ and $(K \cap M)=0$ then $\operatorname{Hom}((M+K) / M, N) \cong \operatorname{Hom}(K, N)$ $\neq 0$ which contradicts $M \triangle N$.

(b) Let $(M \cap L) \leqq K \leqq L$. Then $(M \cap L)=(K \cap M)$. So

$$
\begin{aligned}
\operatorname{Hom}(K /(M \cap L), L) & =\operatorname{Hom}(K /(K \cap M), L) \cong \operatorname{Hom}((K+M) / M, L) \\
& \leqq \operatorname{Hom}((K+M) / M, N)=0 .
\end{aligned}
$$

(e) is easily checked.

(f) Suppose $M \triangle N$. Let $f \in \operatorname{Hom}(I, I)$ such that $f(M)=0$. If $L=f^{-1}(N) \cap N$ then $M \leqq L \leqq N$ and $\left.f\right|_{L} \in \operatorname{Hom}(L, N)$ such that $\left.f\right|_{L}(M)=0$. So $\left.f\right|_{L}=0$, i.e. $0=f(L)$ $=N \cap f(N)$. Hence since $N$ is essential in $I, f(N)=0$.

For the converse let $M \leqq L \leqq N$ and $f \in \operatorname{Hom}(L, N)$ such that $f(M)=0$. Since $I$ is injective there exists $g \in \operatorname{Hom}(I, I)$ extending $f$. Thus $g(M)=0$. It follows from our hypothesis that $g(N)=0$, so $f(L)=0$. Hence $M \Delta N$.

(c) follows easily from (f) since $\mathscr{M}$ has injective envelopes.

(d) follows immediately from (b) and (c).

THEOREM 1.2. Every $M$ in $\mathscr{M}$ has a maximal dense extension $Q(M)$ in $\mathscr{M}$, i.e. an extension $Q(M)$ satisfying the following two equivalent conditions:

(1) $M \triangle Q(M)$ and if $Q(M) \triangle N$ then $Q(M)=N$.

(2) $M \triangle Q(M)$ and if $M \triangle N$ then there exists a unique monomorphism from $N$ to $Q(M)$ extending the identity on $M$.

Thus $Q(M)$ is unique up to unique isomorphism extending the identity of $M$. Moreover, if I is injective envelope of $M$ such that $M \leqq Q(M) \leqq I$ and if $M \leqq N \leqq I$ then $M \triangle N$ if and only if $N \leqq Q(M)$.

Proof. Let $I$ be an injective envelope for $M$.

Let $\Omega=\{f \in \operatorname{Hom}(I, I): f(M)=0\}$.

Let $Q(M)=\bigcap\{\operatorname{Kernel}(f): f \in \Omega\}$. 
Claim 1. $M \triangle Q(M)$ and if $N \leqq I$ such that $M \triangle N$ then $N \leqq Q(M)$ : This follows from 1.1(f).

Claim 2. $Q(M)$ satisfies (2): Let $M \Delta N$ and let $J$ be an injective envelope for $M$ such that $N \leqq J$. Then there is an isomorphism $\phi$ from $J$ to $I$ which extends the identity on $M$. One can easily see that $M \Delta \phi(N)$. So $\phi(N) \leqq Q(M)$. Hence $\phi$ restricted gives a monomorphism $\tau$ from $N$ into $Q(M)$ which extends the identity on $M$. Now suppose $\sigma$ is another monomorphism from $N$ to $Q(M)$ which extends the identity on $M$. Then using the fact that $M \Delta Q(M)$ one sees that the monomorphism $\sigma \tau^{-1}: \tau(N) \rightarrow Q(M)$ must be the inclusion $\tau(N) \leqq Q(M)$. Hence $\sigma=\tau$.

Claim 3. $Q(M)$ satisfies (1): Let $Q(M) \Delta N$ and let $J$ be an injective envelope for $N$. There exists an isomorphism $\phi$ from $J$ to $I$ which extends the identity on $Q(M)$. One can see easily that $M \Delta \phi(N)$. So $\phi(N) \leqq Q(M)$. Hence $N=Q(M)$.

Claim 4: (1) and (2) are equivalent. Let $Q \in \mathscr{M}$. We will say $Q$ satisfies (1) (respectively (2)) if $Q$ satisfies the conditions on $Q(M)$ in (1) (resp. (2)).

Suppose $Q$ satisfies (1). Let $J$ be an injective envelope of $M$ such that $Q \leqq J$. Then there exists an isomorphism $\phi$ from $J$ to $I$ such that $\phi$ extends the identity on $M$. One checks that $M \Delta \phi(Q)$, so $\phi(Q) \Delta Q(M)$ and so $Q=\phi^{-1}(Q(M))$ and $\phi$ restricted is an isomorphism from $Q$ to $Q(M)$ which extends the identity on $M$. Since $Q(M)$ satisfies (2), so does $Q$.

Now suppose $Q$ satisfies (2). If $Q \mathbf{\Delta} N$ then $M \Delta N$ so there exists a unique monomorphism $\phi: N \rightarrow Q$ which extends the identity on $M$. If $i$ is the inclusion $Q \leqq N$ then by the uniqueness of $\phi, \phi=\phi i \phi$. So since $\phi$ is a monomorphism, $i \phi$ is the identity on $N$. Hence $i$ is an isomorphism, i.e. $Q=N$. Thus $Q$ satisfies (1). So we have proved Claim 4.

It is routine to show that if $Q$ satisfies (2) then there is a unique isomorphism from $Q$ to $Q(M)$ which extends the identity on $M$.

The last sentence of the theorem follows from Claim 1 and 1.1(e).

For an open subset $U$ of $X$ we denote the restriction of $A$ to $U$ by $\left.A\right|_{U}$. Likewise for $M$ in $\mathscr{M},\left.M\right|_{U}$ denotes the restriction of $M$ to $U$. Thus $\left.M\right|_{U}$ is an $\left.A\right|_{U}$-Module. We denote by $M_{U}$ the subsheaf of $M$ which restricts to $\left.M\right|_{U}$ on $U$ and 0 on the complement of $U$ in $X$ (cf. [3, pp. 139-140]).

The notation Hom ( , ) will be used both to denote $A$-Module homomorphisms and to denote $\left.A\right|_{U}$-Module homomorphisms depending on the context. Likewise depending on context $\leqq$ and $\mathbf{\Delta}$ will be used to denote subobjects and dense subobjects in both the categories of $A$-Modules and $\left.A\right|_{U}$-Modules. For $M$ and $N$ in $\mathscr{M}$, $\mathscr{H}$ om $(M, N)$ denotes the sheaf of abelian groups given by

$$
\mathscr{H} \text { om }(M, N)(U)=\operatorname{Hom}\left(\left.M\right|_{U},\left.N\right|_{U}\right)
$$

with restrictions as connecting maps (cf. [3, p. 127]).

Proposition 1.3. For $M$ in $\mathscr{M}$ and $U$ an open subset of $X,\left.Q(M)\right|_{U}=Q\left(\left.M\right|_{U}\right)$, i.e. $\left.Q(M)\right|_{U}$ is a maximal dense extension of $\left.M\right|_{U}$ in the category of $\left.A\right|_{U}$-Modules. 
Proof. It is well known that the functor $\left.(~)\right|_{U}$ is exact, preserves essential extensions, injectives, and arbitrary intersections.

Let $I$ be an injective envelope of $M$. Then $\left.I\right|_{U}$ is an injective envelope for $\left.M\right|_{U}$ as an $\left.A\right|_{U}$-Module. Let

and

$$
\Omega=\{f \in \operatorname{Hom}(I, I): f(M)=0\}
$$

$$
\Psi=\left\{h \in \operatorname{Hom}\left(\left.I\right|_{U},\left.I\right|_{U}\right): h\left(\left.M\right|_{U}\right)=0\right\} .
$$

Clearly if $f \in \Omega$ then $\left.f\right|_{U} \in \Psi$. We now show that all the elements of $\Psi$ are of this form. Let $h \in \Psi$. Extending $h$ by zero gives a homomorphism from $I_{U}$ to $I$; this homomorphism annihilates $M_{U}$ so induces a homomorphism $h^{\prime}$ from $I_{U} / M_{U}$ to $I$. Since $M_{U}=\left(M \cap I_{U}\right), I_{U} / M_{U}$ is embedded in $I / M$. So since $I$ is injective there exists $f^{*} \in \operatorname{Hom}(I / M, I)$ such that $f^{*}$ restricts to $h^{\prime}$ on $I_{U} / M_{U}$. Let $f \in \operatorname{Hom}(I, I)$ be the composition of $f^{*}$ with the natural map $I \rightarrow I / M$. Then clearly $f \in \Omega$ and one checks that $\left.f\right|_{U}=h$. Thus $\Psi=\left\{\left.f\right|_{U}: f \in \Omega\right\}$.

Hence

$$
\begin{aligned}
\left.Q(M)\right|_{U} & =\left.(\bigcap\{\operatorname{Kernel}(f): f \in \Omega\})\right|_{U}=\bigcap\left\{\left.\operatorname{Kernel}(f)\right|_{U}: f \in \Omega\right\} \\
& =\bigcap\left\{\operatorname{Kernel}\left(\left.f\right|_{U}\right): f \in \Omega\right\}=\bigcap\{\operatorname{Kernel}(h): h \in \Psi\}=Q\left(\left.M\right|_{U}\right) .
\end{aligned}
$$

Corollary 1.4. If $M \Delta N$ in $\mathscr{M}$ and $U$ is an open subset of $X$ then $\left.\left.M\right|_{U} \Delta N\right|_{U}$ as $\left.A\right|_{U}$-Modules.

Proof. This follows easily from 1.2 and 1.3.

As in the case of rings and modules our main interest is in $Q(A)$ which is called the quotient sheaf of $A$.

LemmA 1.5. As sheaves of abelian groups $\mathscr{H}$ om $(A, Q(A))$ and $\mathscr{H}$ om $(Q(A), Q(A))$ are isomorphic via an isomorphism $\phi$ where for $U$ an open subset of $X$ and $g \in \operatorname{Hom}\left(\left.A\right|_{U},\left.Q(A)\right|_{U}\right), \phi_{U}(g)$ is the unique element of $\operatorname{Hom}\left(\left.Q(A)\right|_{U},\left.Q(A)\right|_{U}\right)$ which restricts to $g$ on $\left.A\right|_{U}$.

Proof. Let $U$ be an open subset of $X$ and $g \in \operatorname{Hom}\left(\left.A\right|_{U},\left.Q(A)\right|_{U}\right)$. By 1.3 and 1.2 we may assume $\left.A\right|_{U} \leqq\left. Q(A)\right|_{U} \leqq I$ where $I$ is an $\left.A\right|_{U}$-injective envelope of $\left.A\right|_{U}$ and $\left.Q(A)\right|_{U}=\bigcap\left\{\operatorname{Kernel}(f): f \in \operatorname{Hom}(I, I)\right.$ and $\left.f\left(\left.A\right|_{U}\right)=0\right\}$. Since $I$ is injective we can extend $g$ to $g^{\prime}$ in $\operatorname{Hom}(I, I)$. Let $f \in \operatorname{Hom}(I, I)$ such that $f\left(\left.A\right|_{U}\right)=0$, then $f g^{\prime}\left(\left.A\right|_{U}\right)=f g\left(\left.A\right|_{U}\right) \leqq f\left(\left.Q(A)\right|_{U}\right)=0$. Hence $g^{\prime}\left(\left.Q(A)\right|_{U}\right) \leqq\left. Q(A)\right|_{U}$. So restricting $g^{\prime}$ we have $g^{\prime \prime} \in \operatorname{Hom}\left(\left.Q(A)\right|_{U},\left.Q(A)\right|_{U}\right)$ such that $g^{\prime \prime}$ restricts to $g$ on $\left.A\right|_{U}$. Moreover, since $\left.\left.A\right|_{U} \triangle Q(A)\right|_{U}, g^{\prime \prime}$ is the unique homomorphism in Hom $\left(\left.Q(A)\right|_{U},\left.Q(A)\right|_{U}\right)$ which extends $g$. So the map $g \mapsto g^{\prime \prime}$ gives a bijection $\phi_{U}$ : Hom $\left(\left.A\right|_{U},\left.Q(A)\right|_{U}\right)$ $\rightarrow \operatorname{Hom}\left(\left.Q(A)\right|_{U},\left.Q(A)\right|_{U}\right)$. Using the uniqueness property it is easy to see that $\phi_{U}$ is a group homomorphism and that $\phi=\left\{\phi_{U}: U\right.$ is an open subset of $\left.X\right\}$ is a sheaf map. Hence $\phi$ is an isomorphism from $\mathscr{H}$ om $(A, Q(A))$ to $\mathscr{H}$ om $(Q(A), Q(A))$. 
Recall that for any $M$ in $\mathscr{M}, \mathscr{H}$ om $(A, M)$ is an $A$-Module and $M$ is $A$-isomorphic to $\mathscr{H}$ om $(A, M)$ via the isomorphism $\mu=\left\{\mu_{U}\right\}$ where for $U$ an open subset of $X$ and $m \in M(U), \mu_{U}(m)$ is the multiplication-like element of $\operatorname{Hom}\left(\left.A\right|_{U},\left.M\right|_{U}\right)$ corresponding to $m$, i.e. for $W$ an open subset of $U$ and $a \in A(W),\left(\mu_{U}(m)\right)_{W}(a)$ $=\left(\rho_{W U}(m)\right) a$ where $\rho_{W U}$ is the connecting map from $M(U)$ to $M(W)$.

THEOREM 1.6. There is a unique ring-sheaf structure for $Q(A)$ which extends the $A$-Module structure on $Q(A)$. With this structure $Q(A)$ is clearly a sheaf of rings extending $A$.

Note. To say that $Q(A)$ has a ring-sheaf structure extending the $A$-Module structure on $Q(A)$ means that for each open subset $U$ of $X, Q(A)(U)$ is a ring with multiplication $Q(A)(U) \times Q(A)(U) \rightarrow Q(A)(U)$ extending the $A(U)$-module operation $Q(A)(U) \times A(U) \rightarrow Q(A)(U)$ and with the same addition as $Q(A)(U)$ has as an $A(U)$-module; and also that the connecting maps of $Q(A)$ as an $A$-Module are ring homomorphisms.

Proof of 1.6. We have that as $A$-Modules $Q(A) \cong \mathscr{H}$ om $(A, Q(A))$ and by 1.5 that as sheaves of abelian groups $\mathscr{H}$ om $(A, Q(A)) \cong \mathscr{H}$ om $(Q(A), Q(A))$. But $\mathscr{H}$ om $(Q(A), Q(A))$ with composition as its multiplicative operations is a sheaf of rings. If we pull this ring-sheaf structure back to $Q(A)$ it requires only a routine check of identifications to see that this ring-sheaf structure extends the $A$-Module structure on $Q(A)$. To see that this ring-sheaf structure is unique we need the following:

Lemma 1.7. If $B$ is a sheaf of rings extending $A$ such that with the induced $A$ Module structure on $B, A \Delta B$, then there exists a unique ring-sheaf embedding of $B$ into $Q(A)$ which extends the identity on $A$.

Proof. We may assume $A \leqq B \leqq Q(A)$ in $\mathscr{M}$. For existence it suffices to show that the ring-sheaf operations on $B$ are just the restriction of those on $Q(A)$. Let $U$ be an open subset of $X$ and let $r \in B(U)$. Define $\mu$ and $\nu:\left.\left.B\right|_{U} \rightarrow Q(A)\right|_{U}$ as follows: for $W$ open in $U$ and $s \in B(W), \mu_{W}(s)=s \circ \rho_{W U}(r)$ and $\nu_{W}(s)=s * \rho_{W U}(r)$ where $\circ$ is the multiplication on $B(W)$ and $*$ is the multiplication on $Q(A)(W)$ and $\rho_{W U}$ is the connecting map from $Q(A)(U)$ to $Q(A)(W)$. Then one checks that both $\mu$ and $\nu$ are $\left.A\right|_{U}$-homomorphisms and that they agree on $\left.A\right|_{U}$. So since $\left.\left.A\right|_{U} \Delta Q(A)\right|_{U}$ we have that $\mu=\nu$. Hence if $s \in B(U)$ we have $s \circ r=s * r$. Thus the $A$-Module monomorphism $B \leqq Q(A)$ is also a ring-sheaf homomorphism; so we have existence. If $\sigma$ and $\tau$ are two ring-sheaf homomorphisms from $B$ to $Q(A)$ which extend the identity on $A$ then $\sigma-\tau$ is an $A$-homomorphism from $B$ to $Q(A)$ and is zero on $A$, so, since $A \triangle Q(A), \sigma=\tau$.

Now to complete the Proof of 1.6 we only have to note that if $Q(A)$ had another ring-sheaf structure which extended the $A$-Module structure on $Q(A)$ then it would follow from 1.7 that there exists an $A$-Module homomorphism from $Q(A)$ to $Q(A)$ 
which extends the identity on $A$ but is different from the identity on $Q(A)$; this would contradict $A \triangle Q(A)$.

Definition. We call the sheaf of rings $Q(A)$ given in 1.6 the quotient sheaf of $A$.

We now turn to Gabriel's thesis [2, p. $365 \mathrm{ff}$.] to get another characterization of $Q(A)$. Let $\mathscr{C}$ be the full subcategory of $\mathscr{M}$ composed of all $M$ in $\mathscr{M}$ having $\operatorname{Hom}(N, A)=0$ for all subobjects $N$ of $M$. It is easy to see that $\mathscr{C}$ is a Serre subcategory of $\mathscr{M}$, i.e. if $0 \rightarrow M \rightarrow L \rightarrow N \rightarrow 0$ is an exact sequence in $\mathscr{M}$, then $L$ is in $\mathscr{C}$ if and only if both $M$ and $N$ are in $\mathscr{C}$. So we can form the quotient category $\mathscr{M} / \mathscr{C}$. One recalls that $\mathscr{M} / \mathscr{C}$ has the same objects as $\mathscr{M}$ and that for $M$ and $N$ in $\mathscr{M} / \mathscr{C}, \operatorname{Hom}_{\mathscr{M} / \mathscr{C}}(M, N)=\operatorname{dir} \lim \operatorname{Hom}_{. /}\left(M^{\prime}, N / N^{\prime}\right)$ where the direct limit is over all subobjects $M^{\prime}$ of $M$ and $N^{\prime}$ of $N$ such that $M / M^{\prime}$ and $N^{\prime}$ are in $\mathscr{C}$. The canonical functor $T: \mathscr{M} \rightarrow \mathscr{M} / \mathscr{C}$ is given by $T(M)=M$ for all $M$ in $\mathscr{M}$ and, for $f \in \operatorname{Hom}_{\mathscr{M}}(M, N), \boldsymbol{T}(f)$ is the image of $f$ in $\operatorname{Hom}_{\mathscr{M} / \mathscr{C}}(M, N)$. It follows easily from [2, Corollary 1, p. 375] that $T$ has an adjoint functor $S$, i.e. that $\mathscr{C}$ is a localizing subcategory of $\mathscr{M}$.

Gabriel shows that $S$ may be defined on objects as follows: If $M$ is in $\mathscr{M} / \mathscr{C}$ then there exists a unique subobject $N$ of $M$ which is maximal with respect to being in $\mathscr{C}$. If $I$ is the injective envelope of $M / N$ in $\mathscr{M}$ then $S(M)$ is the unique subobject of $I$ which is an overobject of $M / N$ and is maximal with respect to having $\boldsymbol{S}(M) /(M / N)$ in $\mathscr{C}$. With such an $\boldsymbol{S}$ we have

Proposition 1.8. $Q(A)=\boldsymbol{S T}(A)$.

Proof. Let $I$ be a fixed injective envelope of $A$ in $\mathscr{M}$. We may assume $A \leqq Q(A)$ $\leqq I$ as in the proof of 1.2 and $A \leqq S T(A) \leqq I$ as above. Since $A$ has no nonzero subobjects in $\mathscr{C}, \boldsymbol{S T}(A)$ is the subobject of $I$ which is maximal such that $\boldsymbol{S T}(A) / A$ is in $\mathscr{C}$. Suppose $f \in \operatorname{Hom}_{\mathscr{M}}(I, I)$ with $f(A)=0$. If $f(\boldsymbol{S T}(A)) \neq 0$ then $f(\boldsymbol{S T}(A)) \cap A$ $\neq 0$. Hence $f$ would induce a nonzero element of $\operatorname{Hom}\left(\left(\boldsymbol{S T}(A) \cap f^{-1}(A)\right), A\right)$ which restricts to zero on $A$. This would contradict the fact that $S T(A) / A$ is in $\mathscr{C}$. Thus $\boldsymbol{S T}(A) \leqq Q(A)$.

On the other hand it is easy to see that since $A \Delta Q(A), Q(A) / A$ is in $\mathscr{C}$. So by the maximality of $\boldsymbol{S T}(A), \boldsymbol{S T}(A)=Q(A)$.

We now define a new sheaf of rings $R=R(A)$ over $X$ as follows: for an open subset $U$ of $X$,

$$
R(U)=\operatorname{dir} \lim \operatorname{Hom}_{A \mid U}\left(D,\left.A\right|_{U}\right)
$$

where the direct limit is over all dense subobjects $D$ of $\left.A\right|_{U}$, and for open subsets $U \subseteq W$ of $X$ the connecting map from $R(W)$ to $R(U)$ is the map induced by the

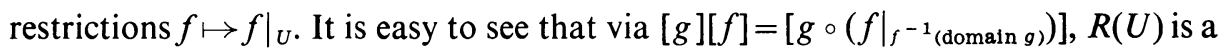
ring and the connecting maps are ring homomorphisms. Also $A$ is naturally embedded in $R$ as a subsheaf of rings via the isomorphism $A \cong \mathscr{H}$ om $(A, A)$.

THEOREM 1.9. $Q(A)$ and $R(A)$ are isomorphic as sheaves of rings via an isomorphism which extends the identity on $A$. 
Proof. Let $U$ be an open subset of $X$. Let $\mathscr{M}^{\prime}$ be the category of $\left.A\right|_{U}$-Modules, $\mathscr{C}^{\prime}$ the full subcategory of all $M$ in $\mathscr{M}^{\prime}$ with $\operatorname{Hom}_{\mathscr{M}}\left(N,\left.A\right|_{U}\right)=0$ for all subobjects $N$ of $M$. Let $\boldsymbol{T}^{\prime}: \mathscr{M}^{\prime} \rightarrow \mathscr{M}^{\prime} / \mathscr{C}^{\prime}$ and $\boldsymbol{S}^{\prime}: \mathscr{M}^{\prime} \mid \mathscr{C}^{\prime} \rightarrow \mathscr{M}^{\prime}$ be the corresponding adjoint functors of Gabriel. Then as abelian groups

$$
\begin{aligned}
Q(A)(U) & \cong \operatorname{Hom}_{\mathscr{M}^{\prime}}\left(\left.A\right|_{U},\left.Q(A)\right|_{U}\right) \\
& \cong \operatorname{Hom}_{\mathscr{M}^{\prime}}\left(\left.Q(A)\right|_{U},\left.Q(A)\right|_{U}\right) \quad(1.5) \\
& \cong \operatorname{Hom}_{\mathscr{M}^{\prime}}\left(S^{\prime} \boldsymbol{T}^{\prime}\left(\left.A\right|_{U}\right), \boldsymbol{S}^{\prime} \boldsymbol{T}^{\prime}\left(\left.A\right|_{U}\right)\right) \quad(1.3,1.8) \\
& \cong \operatorname{Hom}_{\mathscr{M}^{\prime} / \mathscr{C}^{\prime}}\left(\boldsymbol{T}^{\prime} \boldsymbol{S}^{\prime} \boldsymbol{T}^{\prime}\left(\left.A\right|_{U}\right), \boldsymbol{T}^{\prime} \boldsymbol{S}^{\prime} \boldsymbol{T}^{\prime}\left(\left.A\right|_{U}\right)\right) \quad \text { [2, III-2, Lemmas 1, 2] } \\
& \cong \operatorname{Hom}_{\mathscr{M}^{\prime} / \mathscr{C}^{\prime}}\left(\boldsymbol{T}^{\prime}\left(\left.A\right|_{U}\right), \boldsymbol{T}^{\prime}\left(\left.A\right|_{U}\right)\right) \quad \text { [2, III-2, Proposition 3] } \\
& \cong \operatorname{dir} \lim \operatorname{Hom}_{\mathscr{M}^{\prime}}\left(D,\left.A\right|_{U}\right) \quad \text { where the limit is over all }\left.D \Delta A\right|_{U} \\
& =R(U) .
\end{aligned}
$$

Let $\eta_{U}$ be the composition $Q(A)(U) \rightarrow R(U)$. Following Gabriel (in particular the construction of $S$ in the proof of Proposition 4 on p. 372 in [2]) one can check that for $q$ in $Q(A)(U)$, if $\lambda_{q}$ is the multiplication-like element of $\mathrm{Hom}_{\mathscr{M}^{\prime}}\left(\left.A\right|_{U},\left.Q(A)\right|_{U}\right)$ corresponding to $q$ and $\lambda_{q^{*}}$ is the element of $\operatorname{Hom}_{\mathscr{M}^{\prime}}\left(\lambda_{q}^{-1}\left(\left.A\right|_{U}\right),\left.A\right|_{U}\right)$ gotten by restricting $\lambda_{q}$, then $\eta_{U}(q)=\left[\lambda_{q^{*}}\right] \in R(U)$. With this it is easy to see that for each open subset $U$ of $X, \eta_{U}$ is a ring homomorphism and the appropriate connecting diagrams commute, so that $\eta=\left\{\eta_{U}\right\}$ is a ring-sheaf isomorphism from $Q(A)$ to $R$. Also if $U$ is open in $X$ and $a \in A(U) \leqq Q(A)(U)$ then $\eta_{U}(a)=\left[\lambda_{a}:\left.\left.A\right|_{U} \rightarrow A\right|_{U}\right]$ which is the way $A(U)$ is embedded in $R(U)$. So we have that $\eta$ extends the identity on $A$.

2. The sheaves associated with a valuation ring. In this section we start with a valuation ring $V$ which is not a field. We form a sheaf of rings $A_{V}$ corresponding to $V$ and we compute the quotient sheaf $Q\left(A_{V}\right)$. We will see that this quotient sheaf corresponds to the completion of $V$ if $V$ is discrete rank one and that $A_{V}$ is its own quotient sheaf if and only if $V$ is maximal.

For the rest of this section let $V$ be a fixed valuation ring with quotient field $F$ and let $P$ be the maximal ideal of $V$.

Let $X$ be the set of divisors of $V$, i.e. the collection of all ideals of $V$ which are the intersection of principal ideals. For each $x \in X$, let $U_{x}=\{y \in X: x \subseteq y\}$. Topologize $X$ by letting $\mathscr{B}=\left\{U_{x}: x \in X\right\}$ be a basis of open sets for $X$. Since $X$ is linearly ordered by inclusion it is easy to check (cf. [5, p. 28]) that every presheaf of rings or modules over the basis $\mathscr{B}$ gives rise to a unique sheaf over $X$; the structure at the nonbasic open sets is gotten by taking inverse, i.e. projectıve, limits. We will apply this remark repeatedly without further comment by defining sheaves over $X$ and sheaf homomorphisms by only describing their action on the basis $\mathscr{B}$.

We form a sheaf $A_{V}$ over $X$ via $A_{V}\left(U_{x}\right)=V / x$ for all $x \in X$ and for $U_{x} \subseteq U_{y}$ (i.e. $y \subseteq x$ ), the connecting map $\rho_{U_{x} U_{y}}: A_{V}\left(U_{y}\right) \rightarrow A_{V}\left(U_{x}\right)$ is the natural map $V / y \rightarrow V / x$.

The idea of looking at this sheaf $A_{V}$ is due to Harrison. For some idea of why 
$A_{V}$ comes up one might like to look now at 2.7. For our purposes in this paper there are other possibilities for $X$, for example we could have let $X$ be only the principal ideals. However Hoyt Warner's unpublished work on extensions of these sheaves makes the divisorial ideals seem the correct choice.

We will denote $A_{V}$ simply by $A$ when no confusion is likely.

For $x \in X$, we let $W_{x}=\{y \in X: x \varsubsetneqq y\} ; \bigcap W_{x}$ denotes the intersection of all the ideals in $W_{x}$. Thus $\bigcap W_{x} \in X$.

We will see in 2.5 that $Q(A)$ is given by, for $x \in X$,

$$
\begin{aligned}
Q(A)\left(U_{x}\right)=A\left(U_{x}\right) & \text { if } x \neq \bigcap W_{x}, \\
& =A\left(W_{x}\right) \quad \text { if } x=\bigcap W_{x},
\end{aligned}
$$

with connecting maps the appropriate ones from $A$.

It seems certain that there is a simple direct proof that the sheaf of rings defined above is a maximal dense extension of $A$, but $\mathrm{I}$ have not been able to find it. Instead the proof given here depends on 1.9 and requires that we calculate the dense subobjects of the various $\left.A\right|_{U_{x}}$.

Before launching into a series of results which lead up to the calculation of $Q(A)$ in 2.5 , we need to make some easy but useful observations about $X$.

Note 2.1. (a) If $U$ is an open subset of $X$ then either $U=U_{x}$ for some $x$ in $X$ or $U=W_{x}$ for some $x$ in $X$ such that $x=\bigcap W_{x}$.

(b) If $P$ is principal and $x \in X$ and $x \neq V$ then $x=\bigcap W_{x}$ if and only if $x$ is zero or nonprincipal.

(c) If $P$ is not principal then for every $x$ in $X$ with $x \neq V, x=\bigcap W_{x}$.

(d) For any $x$ in $X, \bigcap W_{x}=\bigcap\left\{y \in W_{x}: y\right.$ is principal $\}$.

Proof. One checks (a) and (d).

(b) If $x=0$ then it is easy to see that $x=\bigcap W_{x}$, so we may assume $x \neq 0$. Let $P=p V$. If $x=d V$ then it is easy to see that $x \varsubsetneqq p^{-1} d V=\bigcap W_{x}$. Conversely if $x$ is nonprincipal then clearly $x=\bigcap W_{x}$.

(c) Suppose $P$ is not principal. Let $x \in X$ such that $x \neq V$. Just suppose $x \varsubsetneqq \bigcap W_{x}$. Then $x$ must be principal, say $x=d V$. Choose $a \in\left(\bigcap W_{x}\right) \backslash x$. Then $x \varsubsetneqq a V \subseteq \bigcap W_{\dot{x}}$ which implies that $x \subseteq a P \varsubsetneqq a V$. If $x \varsubsetneqq a P$ then choosing $b \in(a P \mid x)$ would give $x \varsubsetneqq b V \varsubsetneqq \bigcap W_{x}$ which is impossible. So $x=a P$. Hence $P=a^{-1} d V$. Thus $P$ is principal, a contradiction.

We now show that for any $x$ in $X,\left.A\right|_{U_{x}}$ has a unique minimal dense subobject. The description of this minimal dense subobject depends on whether $P$ and/or $x$ is principal. Once we have these minimal dense subobjects, 1.9 will allow us to compute $Q(A)$. Much of the following calculation depends on methods developed by Hoyt Warner.

We consider first the case where $P$ is principal.

Proposition 2.2. Let $P$ be principal and $x$ in $X$.

(a) If $x$ is principal and nonzero then $\left.A\right|_{U_{x}}$ has no proper dense subobjects. 
(b) If $x$ is nonprincipal or zero and $D=\left(\left.A\right|_{U_{x}}\right)_{W_{x}}$, i.e. $D$ is the subobject of $\left.A\right|_{U_{x}}$ given by, for $y \in U_{x}$,

$$
\begin{aligned}
D\left(U_{y}\right) & =0 & & \text { if } y=x, \\
& =V / y & & \text { if } y \in W_{x},
\end{aligned}
$$

then $\left.D \triangle A\right|_{U_{x}}$ and if $D^{\prime}$ is any other dense subobject of $\left.A\right|_{U_{x}}$ then $D \leqq D^{\prime}$.

Proof. (a) This is trivial if $x=V$, so suppose $x \neq V$. Let $P=p V$ and $x=d V$ with $d \neq 0$. Let $E$ be a proper subobject of $\left.A\right|_{U_{x}}$. It is easy to see that this implies $E\left(U_{x}\right)$ $\varsubsetneqq V / x$. Hence $E\left(U_{x}\right) \subseteq P / x$. Define $f:\left.\left.A\right|_{U_{x}} \rightarrow A\right|_{U_{x}}$ via: for $y \in U_{x}$ and $a+y$ in $A\left(U_{y}\right), f_{U_{y}}(a+y)=a d p^{-1}+y$. Clearly this gives an $\left.A\right|_{U_{x}}$-homomorphism $f$ and, for $y \in W_{x}, f_{U_{y}}=0$ since $P d p^{-1}=x \varsubsetneqq y$ implies $V d p^{-1} \subseteq y$. And $f(E)=0$ since $f_{U_{x}}(P / x)$ $=0$. But $f \neq 0$, since $f_{U_{x}}(1+x)=d p^{-1}+x \neq 0$. So $E$ is not dense in $\left.A\right|_{U_{x}}$.

(b) Again let $P=p V$. Let $D \leqq E \leqq\left. A\right|_{U_{x}}$. Let $f:\left.E \rightarrow A\right|_{U_{x}}$ such that $f(D)=0$. Then $f_{U_{y}}=0$ for all $y \in W_{x}$ and if $(a+x) \in E\left(U_{x}\right)$ with say $f_{U_{x}}(a+x)=b+x$, then $b+y$ $=f_{U_{y}}(b+y)=0$ for all $y$ in $W_{x}$. So $b \in \bigcap W_{x}=x, 2.1(\mathrm{~b})$. Thus $f=0$. Hence $\left.D \triangle A\right|_{U_{x}}$.

Now let $\left.D^{\prime} \triangle A\right|_{U_{x}}$. Just suppose $D$ is not a subobject of $D^{\prime}$. Then there exists $z \in W_{x}$ such that $D\left(U_{z}\right) \nsubseteq D^{\prime}\left(U_{z}\right)$, i.e. such that $V / z \nsubseteq D^{\prime}\left(U_{z}\right)$. By $2.1(\mathrm{~b})$, (d) we may assume $z$ is principal, say $z=e V$. Now $V / z \nsubseteq D^{\prime}\left(U_{z}\right)$ implies that $D^{\prime}\left(U_{z}\right) \subseteq P / z$. Let $E$ be the subobject of $\left.A\right|_{U_{x}}$ given by, for $y$ in $U_{x}$,

$$
\begin{aligned}
& E\left(U_{y}\right)=P / y \quad \text { if } x \subseteq y \varsubsetneqq z, \\
& =V / y \text { if } z \subseteq y \text {. }
\end{aligned}
$$

Then $D^{\prime} \leqq E \leqq\left. A\right|_{U_{x}}$. Let $f:\left.E \rightarrow A\right|_{U_{x}}$ be given by, for $y$ in $U_{x}$ and $(a+y)$ in $E\left(U_{y}\right)$,

$$
\begin{aligned}
f_{U_{y}}(a+y) & =0 & & \text { if } x \subseteq y \subsetneq z, \\
& =\left(a e p^{-1}+y\right) & & \text { if } z \subseteq y .
\end{aligned}
$$

Then it is easy to see that $f$ is an $\left.A\right|_{U_{x}}$-homomorphism and that $f\left(D^{\prime}\right)=0$. But $f$ is nonzero since $f_{U_{z}}(1+z)=\left(e d^{-1}+z\right) \neq 0$. This contradicts $\left.D^{\prime} \Delta A\right|_{U_{x}}$.

Proposition 2.3. If $P$ is nonprincipal, $x \in X$ and if $D$ is the subsheaf of $\left.A\right|_{U_{x}}$ given by, for $y \in U_{x}$,

$$
\begin{aligned}
D\left(U_{y}\right) & =0 & & \text { if } y=x, \\
& =P / y & & \text { if } y \in W_{x},
\end{aligned}
$$

then $\left.D \triangle A\right|_{U_{x}}$ and if $D^{\prime}$ is any other dense subobject of $\left.A\right|_{U_{x}}$ then $D \leqq D^{\prime}$. We will denote this $D$ as $\left(\left.P A\right|_{U_{x}}\right)_{\mathrm{W}_{x}}$.

Proof. For $x=V$ the result is trivial so we assume $x \neq V$. To see that $\left.D \triangle A\right|_{U_{x}}$ let $D \leqq E \leqq\left. A\right|_{U_{x}}$ and $f \in \operatorname{Hom}\left(E,\left.A\right|_{U_{x}}\right)$ such that $f(D)=0$. Just suppose $f \neq 0$. If $f_{U_{y}}=0$ for all $y \in W_{x}$ then, by 2.1 (c), $f_{U_{x}}=0$, hence $f=0$, a contradiction. So choose $y \in W_{x}$ such that $f_{U_{y}} \neq 0$. Then $E\left(U_{y}\right)=V / y$ so there exists $c \in V$ such that, for all $a \in V, f_{U_{y}}(a+y)=c a+y$. But $f_{U_{y}}(P / y)=0$. So we have $c P \subseteq y \varsubsetneqq c V$. Since $y$ is a 
divisor we can choose $b \in V$ such that $y \subseteq b V \varsubsetneqq c V$. Hence $P \subseteq c^{-1} b V \varsubsetneqq V$. Thus $P$ is principal, a contradiction. Hence $f=0$ and $\left.D \Delta A\right|_{U_{x}}$.

Now let $\left.D^{\prime} \Delta A\right|_{U_{x}}$. Just suppose $D$ is not a subobject of $D^{\prime}$. Then there exists some $w$ in $W_{x}$ such that $P / w \nsubseteq D^{\prime}\left(U_{w}\right)$. Thus $D^{\prime}\left(U_{w}\right)=\mathfrak{a} / w$ where $\mathfrak{a}$ is an ideal of $V$ such that $\mathfrak{a} \varsubsetneqq P$. Choose $d \in(P \mid \mathfrak{a})$ and $e$ in $(P \mid d V)$ (which is possible since $P$ is not principal). So we have $w \varsubsetneqq \mathfrak{a} \varsubsetneqq d V \varsubsetneqq e V \varsubsetneqq P$. By 2.1(c), (d) there exists a principal ideal $z$ of $V$ such that $x \varsubsetneqq z \varsubsetneqq w$. Note that this implies $e^{-1} z \varsubsetneqq e^{-1} w$ and since $z$ is principal and nonzero, $e^{-1} z \varsubsetneqq d^{-1} z$. So $e^{-1} z \varsubsetneqq\left(e^{-1} w \cap d^{-1} z\right)$. Choose $c$ in $\left(e^{-1} w \cap d^{-1} z\right)$ but not in $e^{-1} z$.

Let $E$ be the subobject of $\left.A\right|_{U_{x}}$ given by, for $y$ in $U_{x}$,

$$
\begin{aligned}
E\left(U_{y}\right) & =D^{\prime}\left(U_{y}\right) & & \text { if } x \subseteq y \subsetneq z, \\
& =e V / y & & \text { if } z \subseteq y \subseteq w, \\
& =V / y & & \text { if } w \subsetneq y .
\end{aligned}
$$

Note that $D^{\prime} \leqq E \leqq\left. A\right|_{U_{x}}$.

Let $f:\left.E \rightarrow A\right|_{U_{x}}$ be the $\left.A\right|_{U_{x}}$-homomorphism given by, for $y$ in $U_{x}$ and $(a+y)$ in $E\left(U_{y}\right)$,

$$
\begin{aligned}
f_{U_{y}}(a+y) & =0 & & \text { if } x \subseteq y \subsetneq z, \\
& =(c a+y) & & \text { if } z \subseteq y \subseteq w, \\
& =0 & & \text { if } w \subsetneq y .
\end{aligned}
$$

Then one checks that $f\left(D^{\prime}\right)=0$. But $f_{U_{z}}(e+z) \neq 0$, so $f \neq 0$. Hence $D^{\prime}$ is not dense in $\left.A\right|_{U_{x}}$, a contradiction.

Lemma 2.4. Let $P$ be nonprincipal, $x$ in $X$, and $D=\left(\left.P A\right|_{U_{x}}\right)_{\mathrm{W}_{x}}$ as in 2.3. Any $f$ in Hom $\left(D,\left.A\right|_{U_{x}}\right)$ can be extended uniquely to $f^{*}$ in Hom $\left(\left(\left.A\right|_{U_{x}}\right)_{W_{x}},\left.A\right|_{U_{x}}\right)$. This gives a bijection ( )* from $\operatorname{Hom}\left(D,\left.A\right|_{U_{x}}\right)$ to $\operatorname{Hom}\left(\left(\left.A\right|_{U_{x}}\right)_{W_{x}},\left.A\right|_{U_{x}}\right)$ for which the following equation makes sense and holds for all $f$ and $g$ in $\operatorname{Hom}\left(D,\left.A\right|_{U_{x}}\right):(f \circ g)^{*}=f^{*} \circ g^{*}$.

Proof. Let $f \in \operatorname{Hom}\left(D,\left.A\right|_{U_{x}}\right)$. Let $w \in W_{x}$. By 2.1(c), (d) we know there is a principal ideal $z=d V$ such that $x \varsubsetneqq z \varsubsetneqq w$. Choose an element $e$ of $P$ such that $z \subseteq e w$. (One such $e$ is gotten by choosing $h \in V$ such that $z \varsubsetneqq h V \varsubsetneqq w$ and letting $e=h^{-1} d$.)

Claim 1. For any $p \in P$ there exists an $a \in V$ such that $f_{U_{z}}(p+z)=(a+z)(p+z)$. This claim is clear if $p \in z$. If $p \notin z$ then $p^{-1} d \in V$. Let $b \in V$ such that $f_{U_{z}}(p+z)$ $=b+z$. Then $0=f_{U_{z}}(d+z)=\left(p^{-1} d+z\right)(b+z)$. So $p^{-1} d b \in d V$. Hence $b \in p V$, which gives the claim.

Claim 2. There is a unique element $(a+w)$ of $V / w$ such that, for all $p$ in $P$, $f_{U_{w}}(p+w)=(a+w)(p+w)$.

Let $a \in V$ such that $f_{U_{z}}(e+z)=(a+z)(e+z)$. Let $p \in P$. If $p V \subseteq e V$ then it is easy to check that $f_{U_{z}}(p+z)=(a+z)(p+z)$, hence $f_{U_{w}}(p+w)=(a+w)(p+w)$. So suppose $e V \subseteq p V$. Let $c \in V$ such that $f_{U_{z}}(p+z)=(c+z)(p+z)$. Then since $e \in p V$ it is easy to check that $f_{U_{z}}(e+z)=(c+z)(e+z)$. Hence $(a-c) e$ is in $z$. This implies that $a-c$ 
is in $w$, for if not then $w \varsubsetneqq(a-c) V$ and so $z \subseteq e w \varsubsetneqq(a-c) e V$, a contradiction. Hence $f_{U_{w}}(p+w)=(c+w)(p+w)=(a+w)(p+w)$. So we have existence. Now suppose that $a^{\prime} \in V$ such that $f_{U_{w}}(p+w)=\left(a^{\prime}+w\right)(p+w)$ for all $p \in P$. If $a^{\prime}+w \neq a+w$ then $w \varsubsetneqq\left(a^{\prime}-a\right) V$ and so (by $\left.2.1(\mathrm{c}),(\mathrm{d})\right)$ there exists $b$ in $V$ such that $w \varsubsetneqq b V \varsubsetneqq\left(a^{\prime}-a\right) V$. Hence $b\left(a^{\prime}-a\right)^{-1} \in P$ and $\left(a^{\prime}+w\right)\left(b\left(a^{\prime}-a\right)^{-1}+w\right)=f_{U_{w}}\left(b\left(a^{\prime}-a\right)^{-1}+w\right)=(a+w)$ $\cdot\left(b\left(a^{\prime}-a\right)^{-1}+w\right)$. Subtracting, we get that $b+w=0$. Thus $b V \subseteq w$, a contradiction. Hence $a^{\prime}+w=a+w$ and we have uniqueness, which completes the claim.

Now for each $w$ in $W_{x}$ let $a_{w}$ in $V$ such that $a_{w}+w$ is the unique element of $V / w$ given in Claim 2. It is easy to see that if $y \subseteq w$ in $W_{x}$ then $a_{w}-a_{y}$ is in $w$. Thus if $f^{*}:\left.\left(\left.A\right|_{U_{x}}\right)_{\mathrm{W}_{x}} \rightarrow A\right|_{U_{x}}$ is defined via for $y \in U_{x}$ and $(r+y) \in\left(\left.A\right|_{U_{x}}\right)_{\mathrm{W}_{x}}\left(U_{y}\right)$

$$
\begin{aligned}
f_{U_{y}}^{*}(r+y) & =0 & & \text { if } x=y, \\
& =\left(a_{y}+y\right)(r+y) & & \text { if } y \in W_{x},
\end{aligned}
$$

then $f^{*}$ is an $\left.A\right|_{U_{x}}$-homomorphism which clearly extends $f$. It is unique since $D$ is dense in $\left.A\right|_{U_{\boldsymbol{x}}}$.

Finally if $f$ and $g$ are in $\operatorname{Hom}\left(D,\left.A\right|_{U_{x}}\right), f \circ g$ makes sense since $\left.g^{-1}(D) \Delta A\right|_{U_{x}}$ so, by $2.3, D \leqq g^{-1}(D)$. It is obvious that $g^{*}\left(\left(\left.A\right|_{U_{x}}\right)_{W_{x}}\right) \subseteq\left(\left.A\right|_{U_{x}}\right)_{W_{x}}$, so $f^{*} \circ g^{*}$ makes sense and using the uniqueness condition it is easy to see that $(f \circ g)^{*}=f^{*} \circ g^{*}$.

Let $S$ be the sheaf of rings extending $A$ given by, for $x$ in $X$,

$$
\begin{aligned}
S\left(U_{x}\right) & =A\left(U_{x}\right) \quad \text { if } x \neq \bigcap W_{x}, \\
& =A\left(W_{x}\right) \text { if } x=\bigcap W_{x},
\end{aligned}
$$

with connecting maps the corresponding ones for $A$. Thus

$$
\begin{aligned}
S\left(U_{x}\right) & =V / x & & \text { if } P \text { is principal and } x \text { is nonzero and principal, } \\
& =\operatorname{proj} \lim V / y & & \text { where the limit is over all } y \text { in } W_{x}, \text { otherwise. }
\end{aligned}
$$

The embedding of $A$ into $S$ is the natural one composed of the identities on $V / x$ when $x \neq \bigcap W_{x}$ and the natural maps $V / x \rightarrow \operatorname{proj} \lim V / y$ when $x=\bigcap W_{x}$.

We now see that $S=Q(A)$ which completes our computation of $Q(A)$.

THEOREM 2.5. $S$ and $Q(A)$ are isomorphic as sheaves of rings via an isomorphism which extends the identity on $A$.

Proof. By 1.9 it will suffice to give an isomorphism from $R(A)$ to $S$ which extends the identity on $A$. We let $R=R(A)$.

Consider first the case where $P$ is not principal. In this case it follows from 2.3 that for any $x$ in $X, R\left(U_{x}\right)=\operatorname{Hom}\left(\left(\left.P A\right|_{U_{x}}\right)_{W_{x}},\left.A\right|_{U_{x}}\right)$ and the ring operation is just composition. Using 2.4 it is easy to see that the map $f \mapsto f^{*}$ is a ring isomorphism from $R\left(U_{x}\right)$ to Hom $\left(\left(\left.A\right|_{U_{x}}\right)_{W_{x}},\left.A\right|_{U_{x}}\right)$. And by elementary sheaf theory (cf. [3, 7.3.1]), $\operatorname{Hom}\left(\left(\left.A\right|_{U_{x}}\right)_{\mathrm{W}_{x}},\left.A\right|_{U_{x}}\right) \cong \operatorname{Hom}\left(\left.A\right|_{\mathrm{w}_{x}},\left.A\right|_{\mathrm{W}_{x}}\right) \cong A\left(W_{x}\right)=S\left(U_{x}\right)$ as groups, and it is clear that these are also ring homomorphisms. So composing we have a ring isomorphism $\mu_{U_{x}}: R\left(U_{x}\right) \rightarrow S\left(U_{x}\right)$. And it is easy to check that this gives a ring-sheaf 
isomorphism $\mu$ from $R$ to $S$. Recalling the way $A$ is embedded in $R(A)$ and $S$, it is easy to see that $\mu$ extends the identity on $A$. So we are done in case $P$ is not principal.

Now consider the case where $P$ is principal. In this case we have by 2.2 that for any $x$ in $X$, if $x \neq \cap W_{x}$,

$$
R\left(U_{x}\right)=\operatorname{Hom}\left(\left.A\right|_{U_{x}},\left.A\right|_{U_{x}}\right) \cong A\left(U_{x}\right)=S\left(U_{x}\right),
$$

where the isomorphism is by $A \cong \mathscr{H}$ om $(A, A)$; and if $x=\bigcap W_{x}$,

$$
\begin{aligned}
R\left(U_{x}\right) & =\operatorname{Hom}\left(\left(\left.A\right|_{U_{x}}\right)_{\mathrm{W}_{x}},\left.A\right|_{U_{x}}\right) \cong \operatorname{Hom}\left(\left.A\right|_{\mathrm{W}_{x}},\left.A\right|_{\mathrm{W}_{x}}\right) \\
& \cong A\left(W_{x}\right)=S\left(U_{x}\right),
\end{aligned}
$$

where the first isomorphism is by elementary sheaf theory (cf. [3, 7.3.1]) and the second is by $A \cong \mathscr{H}$ om $(A, A)$. It is clear that the compositions $R\left(U_{x}\right) \rightarrow S\left(U_{x}\right)$ are ring isomorphisms and it is easy to check that the necessary diagrams commute so we have a ring-sheaf isomorphism $\mu: R \rightarrow S$. Again recalling how $A$ is embedded in $R$ and $S$ it is easy to see that $\mu$ extends the identity on $A$.

Now that we have finally calculated $Q(A)$, we can pretty much read off the results claimed in the first paragraph of this section.

If $V$ is a discrete rank one valuation ring then its completion is $\hat{V}=\operatorname{proj} \lim V / x$ where the limit is over all $x \in X \mid\{0\}$. The map $x \rightarrow x \hat{V}$ is a homeomorphism from $X$ to the space of divisors of $\hat{V}$, so we can identify the space of divisors of $\hat{V}$ with $X$. Forming the sheaf $A_{\hat{v}}$ over $X$ we have that $A_{\vec{v}}$ is a sheaf of rings naturally extending $A=A_{V}$.

Proposition 2.6. If $V$ is a discrete rank one valuation ring with completion $\hat{\nabla}$, then $Q\left(A_{v}\right)=A_{\hat{v}}$.

Proof. In this case $P$ and all the elements of $X$ are principal. So by 2.6, $Q(A)$ is given by, for $x$ in $X$, if $x=0$,

$$
Q(A)\left(U_{x}\right)=A\left(W_{x}\right)=\text { proj } \lim V / y\left(\text { over } y \in W_{0}\right) \cong A_{\hat{v}}\left(U_{x}\right) ;
$$

and if $x \neq 0$,

$$
Q(A)\left(U_{x}\right)=A\left(U_{x}\right)=V / x \cong \hat{V} / x \hat{V} \cong A_{\hat{V}}\left(U_{x}\right) .
$$

And it is easy to see that the connecting maps correspond so $Q\left(A_{V}\right)=A_{\hat{v}}$.

Now we recall that a valuation ring $V$ is called maximal in case $V$ has no proper immediate extensions, i.e. in case any valuation ring properly lying over $V$ has either its value group or its residue-class field properly extending that of $V$. In [6, Theorem 4] Zelinsky showed (with credit to Kaplansky) that a valuation ring $V$ is maximal if and only if for any collection of pairs $\left(e_{\gamma}, E_{\gamma}\right)_{\gamma \in \Gamma}$ where the $e_{\gamma}$ are elements of $V$ and the $E_{\gamma}$ are ideals of $V$ and where $\left(e_{\gamma}-e_{\beta}\right) \in E_{\beta}$ when $E_{\gamma} \subseteq E_{\beta}$, there exists $e \in V$ with $\left(e-e_{\gamma}\right) \in E_{\gamma}$ for all $\gamma \in \Gamma$.

The following result is due to Harrison and is what led to the interest in $A_{V}$ in the first place. 
Proposition 2.7. A valuation ring $V$ is maximal if and only if $A_{V}$ is flabby (i.e. has epic connecting maps).

Proof. Suppose $A=A_{V}$ is flabby. Let $\left\{\left(e_{\gamma}, E_{\gamma}\right)\right\}_{\gamma \in \Gamma}$ as above. We must show that there exists an $e$ in $V$ with $e-e_{\gamma} \in E_{\gamma}$ for all $\gamma$ in $\Gamma$. If there exists $\tau$ in $\Gamma$ such that $E_{\tau} \subseteq E_{y}$ for all $\gamma \in \Gamma$ then $e=e_{\tau}$ works. So suppose no such $\tau$ exists. Let $U=\{x \in X$ : there exists $\gamma \in \Gamma$ with $\left.E_{\gamma} \subseteq x\right\}$, then $U$ is an open subset of $X$. For each $x \in U$, choose $\beta \in \Gamma$ such that $E_{\beta} \subseteq x$ and $a_{x}=e_{\beta}$. It is easy to see that $a_{x}+x$ is independent of the choice of $\beta$ and that, for $x \subseteq y$ in $U, a_{x}-a_{y} \in y$. Now $A(U)=$ proj $\lim V / x$ (over $x \in U)$ and $\left\{a_{x}+x\right\}_{x \in U}$ is an element of $A(U)$. So since $A$ is flabby there exists an $a$ in $V$ with $\left(a-a_{x}\right) \in x$ for all $x$ in $U$. Now if $\gamma \in \Gamma$, choose $\delta \in \Gamma$ such that $E_{\delta} \varsubsetneqq E_{\gamma}$ and then choose a principal ideal $x$ such that $E_{\delta} \subsetneq x \subseteq E$. Then $a-e_{y}=\left(a-a_{x}\right)$ $+\left(a_{x}-e_{\delta}\right)+\left(e_{\delta}-e_{\gamma}\right) \in x+x+E_{\gamma} \subseteq E_{\gamma}$. Hence $e=a$ works and $V$ is maximal.

Now suppose $V$ is maximal. If $U$ is an open subset of $X$ then $A(U)=\operatorname{proj} \lim V / x$ (over $x \in U$ ) and it is easy to use Zelinsky's characterization of maximality to see that $\rho_{U X}$ is onto. Hence $A$ is flabby.

So finally we have

Proposition 2.8. A valuation ring $V$ is maximal if and only if $A_{V}=Q\left(A_{V}\right)$.

Proof. Using the characterization $Q(A)=S$ of 2.5 and recalling the way $A$ is embedded in $S$, it is easy to see that $A=Q(A)$ if and only if the maps $\rho_{\mathrm{W}_{x} U_{x}}$ : $A\left(U_{x}\right) \rightarrow A\left(W_{x}\right)$ are epic for all $x \in X$ with $x=\bigcap W_{x}$. Thus if $A$ is flabby then clearly $A=Q(A)$. Conversely suppose that for all $x \in X$ with $x=\bigcap W_{x}$ we have that $\rho_{W_{x} U_{x}}$ is onto. Then since $\rho_{U_{x} X}$ is onto for all $x$ in $X$ and by 2.1 (a) any open subset of $X$ is either of the form $U_{x}$ or of the form $W_{x}$ where $x=\bigcap W_{x}$, it is easy to see that for any open subset $U$ of $X, \rho_{U X}$ is onto, hence $A$ is flabby. So we are done by 2.7 .

\section{REFERENCES}

1. C. Faith, Lectures on injective modules and quotient rings, Lecture Notes in Math., no. 49, Springer-Verlag, Berlin, 1967. MR 37 \#2791.

2. P. Gabriel, Des catégories abéliennes, Bull. Soc. Math. France 90 (1962), 324-448. MR 38 \#1144.

3. R. Godement, Topologie algébrique et théorie des faisceaux, Actualités Sci. Indust., no. 1252; Publ. Math. Univ. Strasbourg, no. 13, Hermann, Paris, 1958. MR 21 \#1583.

4. A. Grothendieck, Sur quelques points d'algèbre homologique, Tôhoku Math. J. (2) 9 (1957), 119-221. MR 21 \#1328.

5. - - Éléments de géométrie algébrique. I. Le langage des schémas, Inst. Hautes Études Sci. Pıbl. Math. No. 4 (1960), 228 pp. MR 36 \#177a.

6. D. Zelinsky, Linearly compact modules and rings, Amer. J. Math. 75 (1953), 79-90. MR $14,532$.

Department of Mathematics, University of Kentucky, Lexington, Kentucky 40506 